\title{
The Relationship Between the Emission-Line Spectra and X-Ray/UV Continuum ${ }^{1}$
}

\author{
Youjun Lu and Tinggui Wang \\ Center for Astrophysics, University of Science and Technology of China, \\ Hefei, Anhui 230026, China
}

According to photoionization calculations, the broad emission-line (BEL) spectrum depends not only on the physical conditions of line-emitting gas, but also on the spectral shape of incident ionizing continuum, especially from the UV to X-ray. Analysis of emission-line spectra and their correlations with the continuum, therefore, provide a way of probing the anisotropy of ionizing continuum on the BLR scale, which is predicted by current models. Previous works have concentrated on explaining the Baldwin effect, an inverse correlation between equivalent width and continuum luminosity. In this contribution, we present the results of an analysis of $75 \mathrm{AGNs}$ which have well-determined soft X-ray spectral parameters from ROSAT and UV line and continuum measurements.

Table 1. Main Correlations

\begin{tabular}{ccrc}
\hline \hline First Quantity & Second Quantity & \multicolumn{1}{c}{$R_{s}$} & $P_{r}$ \\
\hline$\alpha_{u v x}$ & $M_{a b s}$ & -0.471 & $2.0 \times 10^{-5}$ \\
$\alpha_{u v x}$ & $w_{r}$ ( C IV $)$ & 0.538 & $6.3 \times 10^{-7}$ \\
$\alpha_{u v x}$ & $w_{r}($ Ly $\alpha)$ & 0.467 & $2.4 \times 10^{-5}$ \\
$\alpha_{u v x}$ & C IV $/$ Ly $\alpha$ & -0.546 & $4.0 \times 10^{-7}$ \\
\hline
\end{tabular}

The soft X-ray data are either collected from the literature (Wang et al. 1996 , Siebert et al. 1994, 1995) or retrieved from the ROSAT archive and reduced by using EXSAS. The UV continuum and line fluxes are measured from $I U E$ spectra for 67 objects in the sample in an objective way: UV spectra are first corrected for the Galactic reddening, and the continuum at pseudo-line-free windows is then modeled with a single power-law function. Finally, emission lines are fitted with multiple Gaussian functions. The UV data for the remaining eight objects are taken from Laor et al. $(1994,1995)$. The UV to X-ray spectral index $\left(\alpha_{u v x}\right)$ is defined by a power-law $\left(f_{\nu} \propto \nu^{-\alpha_{u v x}}\right)$ connecting the soft X-rays at $1 \mathrm{keV}$ and UV flux at $1335 \AA$. The average value of $\alpha_{u v x}$ for this sample is 1.46. The spectral indices between $1335 \AA$ and $0.2 \mathrm{keV}$ have also been calculated for comparison. Details will be presented elsewhere ( $\mathrm{Lu}$ et al., in preparation).

\footnotetext{
${ }^{1}$ This work is supported by the National Natural Science Foundation of China.
} 


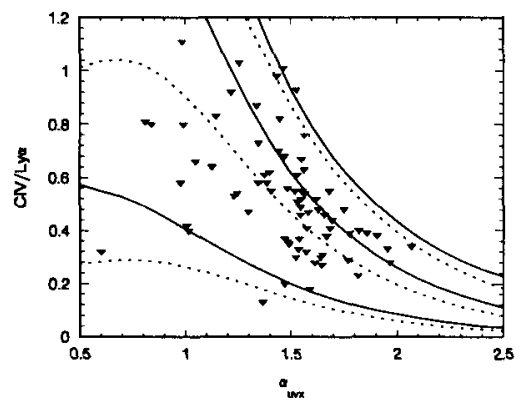

Figure 1. C IV / Ly $\alpha$ flux ratio vs. spectral index $\alpha_{u v x}$. The triangles represent the observational values of the sources. The theoretical lines from photoionization models are plotted; dotted lines and solid lines correspond to hydrogen densities $10^{9} \mathrm{~cm}^{-3}$ and $10^{10} \mathrm{~cm}^{-3}$, respectively. From lower to upper, the three dotted or solid lines correspond to ionizing parameter $U=10^{-2.5}, 0.01$ and 0.1 , respectively.

The results of a Spearman rank correlation analysis are summarized in Table 1. The CIV/Ly $\alpha$ flux ratio, equivalent widths $w_{r}(\mathrm{CIV}), w_{r}(\operatorname{Ly} \alpha)$, and absolute magnitude $M_{a b s}$ are significantly anti-correlated with $\alpha_{u v x}$. The correlations weaken significantly if the spectral indices between $1335 \AA$ and $0.2 \mathrm{keV}$ are used.

Theoretical emission-line spectra are calculated for column densities $10^{9}<$ $n_{\mathrm{H}}<10^{11} \mathrm{~cm}^{-3}$, ionization parameters $10^{-2.5}<U<0.1$, a typical cloud column density $10^{23} \mathrm{~cm}^{-2}$, and solar abundances. The incident ionizing continua are assumed to be power-laws in $\mathrm{UV} / \mathrm{X}$ with slopes covering the observed range, but otherwise an average AGN continuum (Mathews \& Ferland 1987).

Clearly, the correlation between the CIV/Ly $\alpha$ flux ratio and $\alpha_{u v x}$ can be well explained by photoionization models (Fig. 1). It is obvious that the scatter in the CIV/Ly $\alpha$ ratio for constant $\alpha_{u v x}$ can be explained by models with different BLR parameters. The correlations between the equivalent widths $w_{r}(\mathrm{C}$ IV $)$ and $w_{r}(\operatorname{Ly} \alpha)$ and the spectral index $\alpha_{u v x}$ also can be explained by the model under the assumption that the collective covering factor is in the range 0.1-0.25.

Since these results explain the relationships between the BELs and UV/X continua well, we can conclude that the input ionizing continuum is not very different from the observed continuum, i.e., there is no serious anisotropy of the AGN continuum emission on BLR scales.

\section{References}

Brinkmann, W., \& Siebert, J. 1994, A\&A, 285, 812.

Brinkmann, W., Bergeron, J., \& Siebert, J. 1995, A\&A, 293, 985.

Laor, A., et al. 1994, ApJ, 420, 110.

Laor, A., et al. 1995, ApJS, 99, 1.

Mathews, W. G., \& Ferland, G. J. 1987, ApJ, 323, 456.

Wang, T., Brinkmann, W., \& Bergeron, J. 1996, A\&A, 309, 81. 